International Review of Research in Open and Distributed Learning Volume 16, Number 5

\title{
Public Response to "the MOOC Movement" in China: Examining the Time Series of Microblogging 1
}

Jingjing Zhang, Kirk Perris ${ }^{2}$, Qinhua Zheng and Li Chen

Big Data Centre for Technology-Mediated Education, Research Centre of Distance Education, Beijing Normal University, China

\begin{abstract}
In China, microblogging is an extremely popular activity and is proving to be an effective mechanism to gauge perceptions about social phenomena. Between 2010 and 2015 Sina Weibo, China's largest microblogging website, generated 95,015 postings from 62,074 users referencing the term massive open online courses (MOOCs), a method of online course delivery popularized in North America that has spread globally. Time series analyses revealed distinct patterns in the volume of postings during a four-year period, and subsequently by month, by week, and by the time of day. The volume of postings during the week, for example, peaked on Monday and declined daily to a low point on Saturday. Relative to maximizing learner engagement, the findings may provide insight to parties who deliver MOOCs to employ or test strategies on timing (i.e., time of year to offer/not offer a MOOC, time of week to release/not release new material, time of day to schedule/not schedule chat sessions). The paper also serves to demonstrate a mechanism to retrieve big data from social media sources, otherwise underutilized in educational research.
\end{abstract}

Keywords: MOOCs; microblogging; big data; time series; Sina Weibo

\section{Introduction}

\section{MOOCs are no longer a North American phenomenon}


MOOCs are no longer a North American phenomenon. Since 2011, when Stanford University decided to offer several reputable courses online for free, MOOCs have expanded to engage learners around the world. The momentum is far greater than reported, with overseas learners enrolling in a MOOC at a preferred North American institution (Universities UK, 2013). Institutions in at least 50 countries offer a MOOC (MOOC List, 2014), most commonly in partnership with a MOOC provider, such as Coursera or edX. The global appeal of institutions to design MOOCs domestically suggests there is a desire from learners for greater contextualization (e.g., curriculum, language, culture) that the more popular MOOCs in North America do not provide. The major challenge facing other providers of MOOCs is the persistent draw to the likes of Stanford or Harvard - unmatched in terms of prestige and perception of quality learning. Newer MOOC providers that are situated outside of North America should continue to leverage their comparative advantage of contextualizing higher learning, but also engage more deeply into researching the behaviour of online learners. The big data that can be mined from MOOCs is invaluable, but underutilized. Considering the socio-economic returns on higher education, MOOCs present an alternative pathway for learning, particularly under conditions around the world where unmet demand persists.

This article aims to offer some empirical evidence relative to time series behaviour of prospective MOOC learners. The findings may be of value to prospective providers of MOOCs who are interested in utilizing time-oriented strategies to augment learner engagement online.

\section{MOOCs in China: A unique context for delivery}

Outside of North America, Chinese universities have been among the most active adopters of MOOCs. Like the immense interests in rankings and research output (i.e., publishing in high impact factor journals), the attention and resources devoted to MOOCs is, in part, a product of following western trends. Yet, like other imported technological trends, Chinese institutions have proven effective at altering phenomena with the infusion of Chinese characteristics. The product is altogether different, or even superior, as in the case of Alibaba (based on eBay) or Sina Weibo (based on Twitter). MOOCs have yet to follow a similar trend, but the social conditions in China seem highly favourable to utilize these learning tools in novel ways.

Among the many social metrics where China is atop, it is home to the world's largest internet population, at 640 million (Jacquot, 2015), and the world's largest higher education population, at 34 million (Ministry of Education, 2010, p. 22). Considering that holders of a university degree have shown to be the most active demographic enrolling in MOOCs elsewhere (Jordan, 2014) the appetite to enrol in MOOCs among China's educated is potentially big. A large proportion of MOOCs is offered by the country's top universities. The likes of Peking University and Tsinghua University have higher rejection rates for campus-based programs than Harvard or MIT (Wong, 2012). Their MOOCs present an opportunity that has been otherwise non-existent. Those who have completed some form of higher learning also have experience learning at an advanced level, increasing the chance of adaptation to an online learning environment. China's objective to create 
a lifelong learning society over the next decade may also benefit from MOOCs as a means to reach the masses with some form of quality higher education.

Another segment of the population where MOOCs may prove beneficial is adult learners with little or no exposure to higher education. According to the National Outline for Medium and Long-term Education Reform and Development (2010-2020), the government aims to reach enrolment of 350 million people into higher education or adult education by 2020 (Ministry of Education, 2010, p. 22). Campus-based solutions are clearly impractical opening up the possibilities for alternative pathways such as online learning that may include MOOCs.

\section{The MOOC landscape in China}

There are several prominent MOOC providers in China, namely, XuetangX, CNMOOC and iCourse163, which have links with edX and Coursera, respectively. Some institutions, such as Beijing Normal University, are offering MOOCs independently. The majority of these MOOCs are designed and delivered in Chinese; some are in imported from abroad and modified with Chinese subtitles or translations.

The research on MOOCs in China has generally focused on two aspects: 1) introductory studies on the MOOC curriculum model and definitions, characteristics, application modes, and case studies. For example, Li and Wang (2012) introduced cMOOCs as a connectivism approach of learning. Wang, P. (2013) talked about cMOOCs and xMOOCs, illustrating the new development and application of massive online open courses. 2) the impact of MOOCs on the reform of traditional classroom instruction and higher education. For example, Wang, W. (2013) talked about the opportunities and challenges that higher education may face as MOOCs are becoming increasingly popular worldwide. Zhang, Hong \& Wen (2013) talked about the emergence of MOOCs from open courseware that allowed more space for learner-centred education, and affected the development of open universities in China.

Amidst the attention paid to MOOC providers and particular case studies from individual MOOCs in China, little focus has been directed towards aggregate data that may offer insights into the behaviour of learners enrolled in a MOOC. This is a reflection of both the infancy of research on MOOCs in China, and the challenge of acquiring adequate or big data to make informative generalizations.

Relative to this paper is the subject of time. Although MOOCs are characterized as being time independent, this is not always the case, nor is it always an appealing feature. Providing start and end dates to a MOOC remains the most common form of scheduling, and synchronous chat sessions between instructors and learners is extremely popular. The classroom culture, to which nearly all learners are accustomed, is often sought in some form when learning online. Although the flexibility of online learning is a draw for prospective learners, some consistency around timing may minimize isolation and attrition both of which characterize the darker side of MOOCs. That said, it may be worth probing some questions around time and MOOCs. For example, are there certain months of the year that are favourable times to deliver a MOOC? Are there certain 
days of the week where learners more frequently access a MOOC? Is there a time of day when learners are most engaged? And what sort of logistical or pedagogical adjustments can be made, if time patterns exist? Considering that learners who enrol in MOOCs are highly heterogeneous (i.e., international enrolment, time zones differences, varying language abilities), such analyses prove challenging, yet if some of the challenges on time could be neutralized, there may be potential to create more favourable learning conditions in MOOCs.

Consider Chinese MOOCs. There is a high degree of uniformity with respect to learners who enrol in such MOOCs. They are predominantly located in $\mathrm{China}^{3}$, speak Chinese, and are immersed in its culture. Few MOOCs can be considered as contextualized to a learning population. Linking the geographical uniformity of enrolment to the topic of time may reveal some commonalities. After all, annual holiday periods, work weeks and time zones are identical across the country. Capturing such information in aggregate has become increasing possible through harnessing big data on social media, and in particular, social networking platforms.

\section{Sina Weibo: China's most popular microblogging site}

In China, social networking is extremely popular. The microblogging platform Sina Weibo (微博) in particular, enables users to engage on a range of issues with other users. The context is unfiltered, and subject only to periodic interference. No wonder there are over 600 million accounts, of which 30\% are monthly users (Smith, 2015). Like Twitter, Sina Weibo limits a posting to 140 characters. Other common functionalities include initiating, re-posting, or responding to other users' postings.

Organizations, corporations and governments in China utilize Sina Weibo to interact with the wider usership. Newscasts often share with its viewers individuals' unsolicited postings reacting to varying social events. This adds a layer of authenticity and immediacy to reporting. Postings in aggregate also create a system of self-regulation. Inaccuracies, for example, are quickly weeded out from trending conversations. Repeat offenders are subject to scorn, and the permanence of the Internet leaves little room to erase an errant posting.

Because of the big data that is readily available, microblogging has presented opportunities to conduct varied research. One widely cited example was a study by Bollen, Mao and Zeng (2010). They conducted a study that forecasted trends in the stock market by analyzing user behaviour on Twitter. They concluded that large volumes of tweets, as postings are called on Twitter, could be used to predict changes in the US stock market. Other studies have shown predictability in the transmission of infectious diseases (Sadikov, Medina, Leskoven \& Garcia-Molina, 2011), a range of social events in China (Guan et. al, 2014).

In relation to education, in researching of Twitter data used in diverse disciplines, Zimmer \& Proferes (2013) conducted a systematic analysis of academic research that relies on the collection and use of Twitter data, creating a topology of Twitter research. Their research reveals that only 13 journal articles used Twitter data in education between 2008 and 2012. Compared to the total number of 379 articles published in other disciplines during the same period of time, educational 
research was lagging behind in that the Twitter data has not received the considerable attention it merits. Overall, there is a lack of research using microblogging data to predict the trend of educational movements. The majority of research views Twitter as a learning tool promoting social interactions among students. Gao, Luo and Zhang (2012) conducted a critical analysis of research on microblogging in education, and illustrated that microblogging in education was mainly utilized as an educational tool to extend learning beyond the classrooms and blurs the line between formal and informal learning. As for MOOCs, microblogging sites (such as Twitter and Sina Weibo) act as an alternative to discussion forums and are used for a large number of MOOC courses. For example, the course "e-Learning and Digital Culture" offered by the University of Edinburgh used Twitter hashtags to create learning activities for their students. Van Treeck and Ebner (2013) also studied two consecutive MOOCs to explore the usefulness of Twitter for learning in massive communities.

In this paper, a time series analysis is provided over two periods: from 2010 to 2015 and 2013 to 2015. The purpose of the paper is to provide an analysis of data referencing MOOCs on Sina Weibo, a popular Chinese microblogging website. The aim is to provide insights that may be transferrable to learner behaviour in a MOOC. Another aim is to demonstrate the utility of data access through social media to inform theory or practice in the learning sciences.

\section{Methods}

In an attempt to study the public response to MOOCs in China, Sina Weibo data that made reference to MOOCs was analyzed. The microblogging site is the most popular of its kind in China. As noted in the previous section there are over 600 million registered accounts and approximately $30 \%$ of accounts are active monthly users who create a high volume of usergenerated content. As Sina Weibo has been used to gauge public perceptions about varying social phenomena it was deemed a valuable data source to conduct longitudinal data mining analyses about MOOCs.

To gather data, a posting was selected if it contained the keyword "MOOC" or "Muke" (慕课). For simplicity in this paper, the phrase MOOC postings includes either of these keywords. A web crawler was utilized to compile the postings. In total, 95,015 postings published by 62,074 users were retrieved on Sina Weibo from September, 2010 to January, 2015. The time series patterns of MOOC postings was analysed using R programming language. Four time frames were selected to analyse the volume of postings: by year, by month, by day of the week, and by the time of day.

It is important to note that the content of the postings do not necessarily reference an individual's experience of learning in a MOOC. Such content analysis is the basis for future research. Instead, the data reveals a set of time frames of MOOC postings. The results may provide insights for prospective designers of MOOCs on strategies to maximize engagement with learners. If, for example, there is a high volume (or low volume) of postings during a particular time of year, providers or designers of MOOCs may consider time-sensitive strategies in delivering a MOOC. The assumption is that purposeful time-sensitive interventions may impact learner engagement, and ultimately enhance learning and minimize attrition. 


\section{Results}

The results are divided into two sections. The first section presents an overview of the patterns of postings on Sina Weibo from 2010 to 2015. The second section presents a time series analysis of postings by month, by day of the week, and by the time of day.

\section{Part One: Public attention towards MOOCs surges in 2013}

During the period of 2010-2015, an increasing number of postings including the term "MOOC," or “Muke/慕课” were posted on Sina Weibo. The volume of postings rose rapidly from only seven in 2010 to 62,162 in 2014 (see Table 1).

Table 1: MOOC postings on Sina Weibo: 2010-2015

\begin{tabular}{|l|l|l|l|l|l|}
\hline & 2010 & 2011 & 2012 & 2013 & 2014 \\
\hline The number of new MOOC postings & 7 & 76 & 944 & 28,160 & 62,162 \\
\hline $\begin{array}{l}\text { The number of new users who } \\
\text { contributed MOOC postings }\end{array}$ & 7 & 60 & 669 & 19,678 & 39,490 \\
\hline
\end{tabular}

The largest jump in MOOC postings was in 2013 when 28,160 appeared on Sina Weibo. In fact, 99\%, or 93,988 MOOC postings, were published from 2013 to 2014, inclusive. China's two preeminent universities, Tsinghua University and Peking University offered their own MOOCs in 2013, suggesting that the surge in MOOC postings was in response to the actions of these two universities. Another possibility was the media coverage of MOOCs that swelled after an infamous New York Times article which hailed 2012 as the year of the MOOC (Pappano, 2012). This article, however, was in response to the immense media attention directed towards MOOCs in the US throughout 2012. Since little MOOC postings on Sina Weibo appeared in 2012, it seems more plausible that domestic activity of MOOCs was the cause of the greater activity on Sina Weibo. A content analysis planned for future research may better illuminate the source of the surge of MOOC postings in 2013.
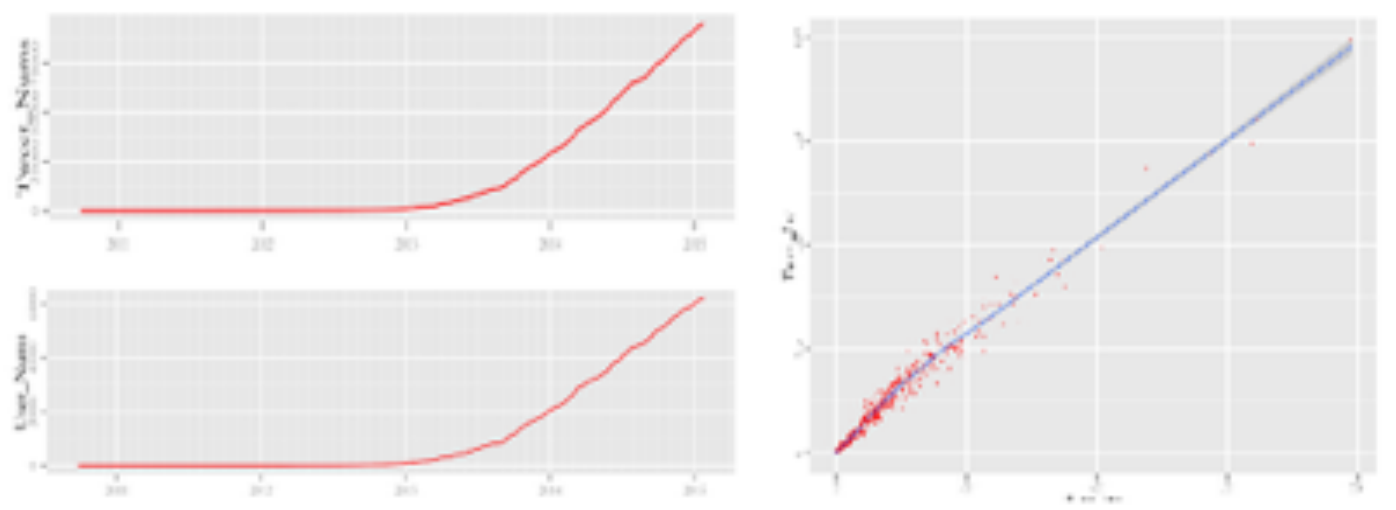

Figure 1: The number of MOOC postings and the number of users contributing MOOC postings: 2010-2015 
One anomaly worth noting is the number of MOOC postings that occurred in 2010 and 2011. It was not until fall 2011 when headlines surfaced about the Stanford MOOCs. Probably the 76 MOOC postings observed in 2011 are linked to this occurrence. Yet it fails to explain the seven MOOC postings in 2010. As noted in the Methods section, a content analysis for future research will be conducted and include this particular anomaly.

As can be observed in Figure 1, the volume of postings that appeared on Sina Weibo coincided with the volume of users contributing MOOC postings, which reached nearly 50,000 users by the beginning of 2015. The majority contributed only one posting and a handful of users contributed 100 or more MOOC postings.

In Table 2, the distribution of number of postings by number of users is displayed. The data demonstrates the volume of MOOC postings by groupings of users

Table 2: The category of users measured by the number of tweets published.

\begin{tabular}{|l|l|l|l|l|l|}
\hline Group & $\begin{array}{l}\text { Number of } \\
\text { tweets }\end{array}$ & $\begin{array}{l}\text { Number of } \\
\text { users }\end{array}$ & Percentage (\%) & $\begin{array}{l}\text { Accumulated } \\
\text { percentage (\%) }\end{array}$ & $\begin{array}{l}\text { Accumulated } \\
\text { number of users } \\
\text { (sorted) }\end{array}$ \\
\hline Grp1 & {$[1-2)$} & 49699 & 82.964 & 82.964 & 59904 \\
\hline Grp2 & {$[2-3)$} & 5996 & 10.009 & 92.973 & 10205 \\
\hline Grp3 & {$[3-4)$} & 1877 & 3.133 & 96.106 & 4209 \\
\hline Grp4 & {$[4-5)$} & 791 & 1.320 & 97.426 & 2332 \\
\hline Grp5 & {$[5-10)$} & 1022 & 1.706 & 99.132 & 1541 \\
\hline Grp6 & {$[10-20)$} & 328 & 0.548 & 99.680 & 519 \\
\hline Grp7 & {$[20-30)$} & 81 & 0.135 & 99.815 & 191 \\
\hline Grp8 & {$[30-40)$} & 42 & 0.070 & 99.885 & 110 \\
\hline Grp9 & {$[40-50)$} & 17 & 0.028 & 99.913 & 68 \\
\hline Grp10 & {$[50-60)$} & 20 & 0.033 & 99.946 & 51 \\
\hline Grp11 & {$[60-70)$} & 4 & 0.007 & 99.953 & 31 \\
\hline Grp12 & {$[70-80)$} & 6 & 0.010 & 99.963 & 27 \\
\hline Grp13 & {$[80-90)$} & 6 & 0.010 & 99.973 & 21 \\
\hline Grp14 & {$[90-100)$} & 0 & 0.000 & 99.973 & 15 \\
\hline Grp15 & {$[100+)$} & 15 & 0.025 & 99.998 & 15 \\
\hline & & & & \\
\hline
\end{tabular}


From Table 2 it can be gleaned that a clear majority of users $(n=49,699)$ contributed only one or two postings. This inverse relationship whereby fewer users contribute more postings is fairly consistent across the 15 groups. The main outlier is group five. There are 1,022 users who fall in this group characterized as contributing six to ten MOOC postings, as compared to 791 users contributing four to five postings. Only 15 individuals contributed over 100 postings in the four year time series.

\section{Part Two: Time series of postings including the term MOOCs on Sina Weibo (2012-2015)}

\section{The summer vacation?}

For the time series analysis, postings were included from 2012 to $2015^{4}$. The bar chart (Figure 2, top) represents the total number of MOOC postings contributed each month. Over the three-year time frame the total number of MOOC postings declines at two intervals each year: January or February and in August. The number of MOOC postings rises sharply in the following month of the given interval, and usually in March or in September.

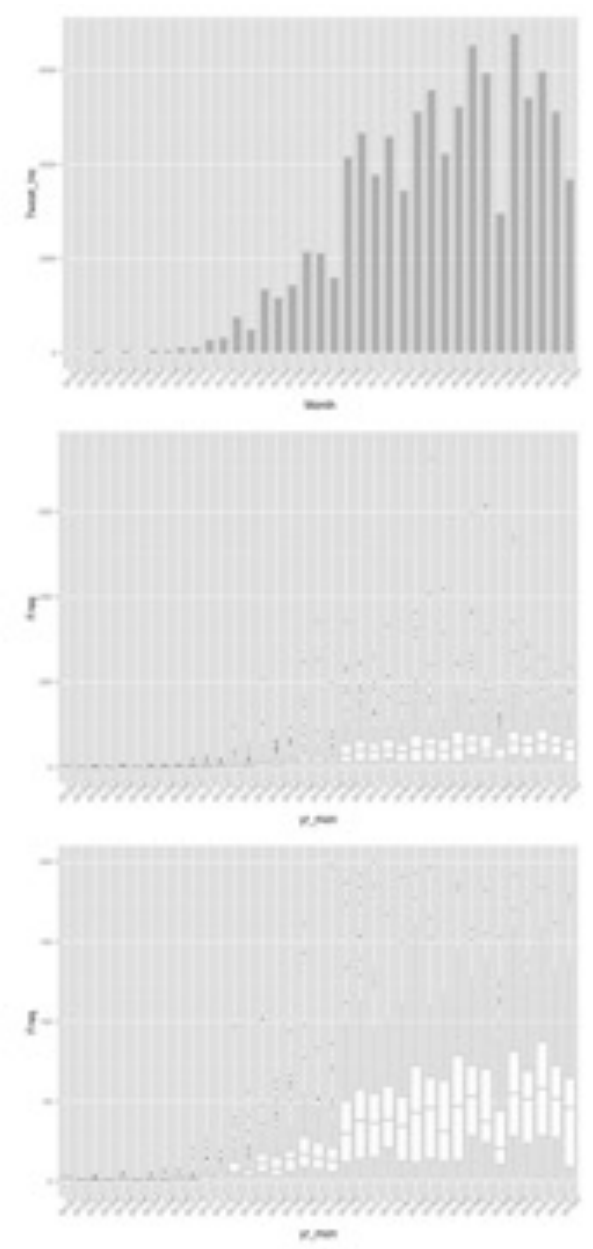

Figure 2. The Monthly Distribution of Postings between 2012 and 2014. 
In Figure 2 (middle), there are a large number of outliers, which potentially affect the mean/sum distribution of tweets. The boxplot distribution (Figure 2, bottom) illustrates the significant difference between the monthly distributions. Like Europe and North America, the timeframe of the education system in China has a winter and a summer break. The posting behaviour of users on Sina Weibo reflects this pattern - postings noticeably drop during these monthly intervals mirroring the two periods in a calendar year when many Chinese take vacation.

Another factor at play may be the availability of MOOCs. Since MOOCs are offered predominantly during the conventional school year (i.e., September to May/June), the lower volume of postings observed in August may be a function of fewer MOOCs that are available online. A search of MOOCs offered by varied platforms that was conducted in January, 2015, supports this argument. On XuetangX all 31 MOOCs began in September and October, 2014. For illustrative purposes, of the 62 English language MOOCs offered by Futurelearn (United Kingdom MOOC provider) in 2014, 23 began in January and February, and 29 were offered in September and October. None were scheduled in July or August. The slowdown in the summer was also found on EdX.

It is unclear whether the drop in MOOC postings observed in the summer is a function of fewer users contributing MOOC postings, or if there are fewer MOOCs being offered (or both). If the former, then MOOC providers should perhaps proceed cautiously if considering offering a MOOC during the Chinese summer break. Less likely is that it may be an opportune time to begin a MOOC. Work and school obligations generally decline in July and August freeing up time to engage in other activities such as enrolling in a MOOC.

There is one other conclusion to be drawn from this inactivity observed on Sina Weibo during the summer months. MOOC postings that were contributed during other times of the year were likely about particular MOOCs, rather than reporting about research, media reports, etc. This assumption relies on there being some other activity about MOOCs during the summer months, such as news reports or research findings, which do not trend on Sina Weibo.

\section{The routine of MOOC postings contributed during the week}

As can be observed in Figure 3, there is a consistent pattern of MOOC postings in a given week. Of the nearly 100,000 postings on Sina Weibo, the number of postings published during a given day during the week (i.e., Monday to Friday) is 1.3 times greater than the number of postings published during a given day during the weekend (i.e., Saturday or Sunday). Further, it is apparent that users on Sina Weibo contributed postings on MOOCs most frequently on Monday and tend to contribute noticeably fewer postings after Wednesday. The volume of postings picks up again on Sunday. The higher volume of postings on Monday is somewhat surprising. Monday can be characterized as a comparatively busy day at work, or school, following the weekend. The fact that users on Sina Weibo are actively contributing postings about MOOCs on this day may suggest individuals have greater amounts of energy to devote to learning. As previously noted, this also assumes that the postings in question are focused on learning, rather than simply sending postings that include comments about MOOCs, or re-postings of original postings. 
Another notable finding is the lull in MOOC postings contributed on Saturdays. Observing patterns of behaviour in a MOOC during a given week may reveal similar findings. In any event, tracking user behaviours in a given week may reveal particular patterns that impact individuals' learning experience in a MOOC.

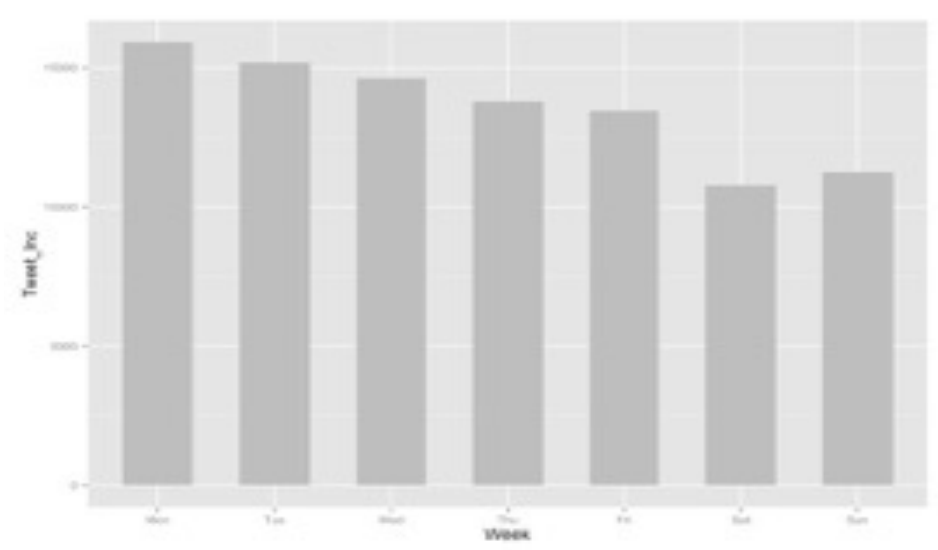

Figure 3. The weekly distribution of postings that include the term MOOC on Sina Weibo.

\section{Contributing postings after work}

Like the patterns observed during the year and during the week, MOOC postings contributed during the day revealed that certain time intervals were more popular than others (See Figure 4). Users contributed postings most frequently in the later evening followed by mid-afternoon and late morning. The comparatively high volume of MOOC postings contributed during after-work hours is not surprising as this is leisure time for many individuals. It also suggests that a significant number of users on Sina Weibo who contributed MOOC postings were working adults. Another popular time to contribute MOOC postings, however, occurs during the workday. It may be inferred that there are a significant number of users who are not working adults, but those who work unconventional hours or have greater flexibility in the day such as students, retirees, or stayat-home caregivers. After all, the ease of submitting a posting to a microblogging site is highly convenient given the ubiquity of smart phones. One last notable finding was that the volume of MOOC postings dipped during the conventional times of eating (particularly lunch and dinner) and sleeping. 


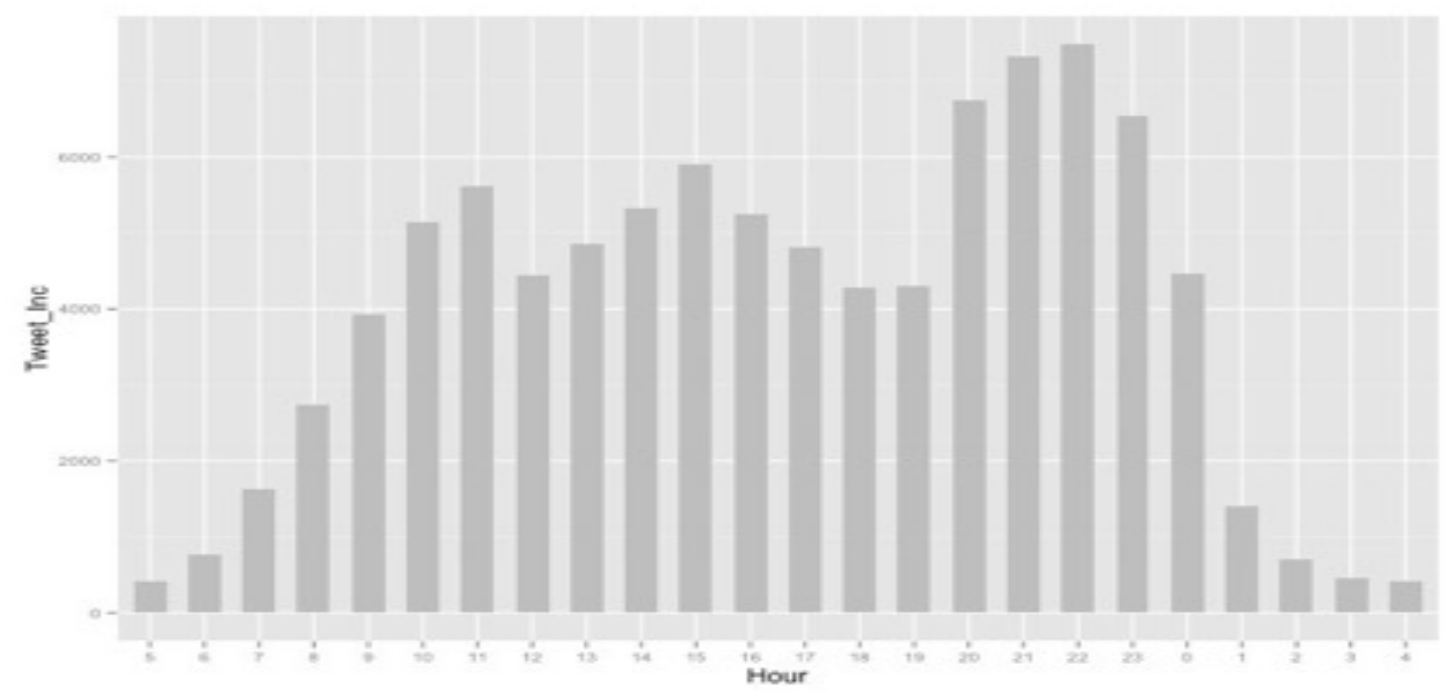

Figure 4. The daily distribution of MOOC postings.

Overall, MOOC postings on Sina Weibo revealed some consistent patterns in four distinct time frames between 2010 and the beginning of 2015. At yearly intervals MOOC postings peaked in 2013 and 2014, times when the buzz around MOOCs became a domestic phenomenon. Within a given year, it was found that around Chinese New Year and summer the number of MOOC postings dropped significantly. No definitive conclusions could be drawn. During the week the volume of MOOC postings reached a high point on Monday and gradually declined to Saturday before picking up again on Sunday. Peak times during the day were later in the evenings, followed by mid afternoon and late morning. In the following section, further inferences are drawn from the findings presented in the results section.

\section{Discussion}

\section{MOOCs for the Chinese Society}

Worldwide interest in MOOCs was stimulated by Stanford's artificial intelligence course, which attracted over 160,000 students from more than 190 countries in the fall of 2011 (Barnes, 2013). Since, several thousand MOOCs have been designed and delivered. Many remain active and new courses and new users are joining the MOOC space daily.

The appeal to MOOCs seems rooted in a cost-effective alternative to conventional higher education. MOOCs are effectively free, and many top universities are partaking in the design and delivery of these large online courses. The appeal is multifaceted. Optics suggest the institution designing and delivering the MOOC is trendy, pedagogically sound in regards to online learning, and engaged in some form of community service. It has also proven to be an excellent mechanism to promote a professor, a faculty, or, ideally, an entire institution. 
In China, there are additional forces at play. There is an immense desire for quality higher education, particularly since the playing field is so uneven. A handful of institutions are recipients of enormous government funding, relegating a large majority of institutions to the margins. The growing draw to MOOCs seems to be linked to the desire to enrol in the country's top universities which are highly selective in their campus-based programs. Like the draw to Stanford or Harvard, an underlying assumption is that China's elite institutions provide quality instruction. If these institutions do not offer some viable credential that is recognized by government and by industry, incentives to enrol in MOOCs will wither. Other potential pathways are to utilize MOOCs to improve teaching domestically, much like the Chinese national top level course project (jingpinkecheng, “精品课程”) aimed to achieve starting in 2003 (Haklev, 2010).

The other big potential for using MOOCs in China is to support a lifelong learning society, a primary objective identified nearly 20 years ago in the Action scheme for invigorating education towards the 21st century (CERNET, 1998). This objective was renewed in 2009 when the government released the National Outline for Medium and Long-term Education Reform and Development (2010-2020). The current objective is for 350 million people in China to have acquired some form of continuing education by 2020. To reach such a target, conventional campus-based solutions are inadequate, even if each of China's 3,000 higher education institutions were running classes 365 days per year. As a delivery mechanism, MOOCs present one potential pathway to reach mass learning populations, particularly since there is mass access to the internet in China. Yet, as has been partially addressed in this paper, learning behaviour needs to be considered in the design and delivery of any learning activity. This is amplified by online learning, whereby the learner is relatively isolated, and located in a learning context that is counterintuitive to the structured classroom that is universally institutionalized.

MOOCs have grown rapidly in China, yet understanding learning behaviour online remains at an early stage of research. Data is limited, rendering generalizations about MOOCs inadequate. One data source that can be harnessed over time is microblogging. It has proven to be an authentic and legitimate source of information sharing that is self-governed by millions of users. In this investigation Sina Weibo, the highly popular microblogging platform, was utilized to compile time series trends about MOOC postings over a four-year period.

MOOC postings on Sina Weibo surged in 2013, after several reputable institutions delivered the first MOOCs in China. In 2014, two prestigious universities based in Shanghai, Fudan University and Shanaghai Jiaotong University followed, and partnered with Futurelearn to release their own MOOC courses through this British-based MOOC provider. Since, more universities have designed their own MOOCs with many having joined MOOC providers. Whether the momentum has continued through Sina Weibo remains to be seen. The peak of activity on Sina Weibo was in 2013, with a drop in the volume of postings in 2014. Although new data has not been compiled, some inferences are offered.

The surge in activity on Sina Weibo regarding MOOCs is comparable to many topics that trend and eventually fade. How far MOOC postings will continue to populate the popular microblogging 
site will depend on several factors. The 600 million+ users on Sina Weibo is a sound indicator of the platform's popularity and its usability. Yet, it operates within a highly competitive social networking milieu. Along with other microblogging platforms, there is QQ and WeChat, both of which have users that measure in the hundreds of millions in China. Although functionality varies between platforms, the premise of connecting innumerable users online is common, and therefore transferrable to varying learning contexts that use MOOCs (Zhang, 2015). Assuming there is a link between the learning experience in MOOCs and MOOC postings on social media, how MOOC designers embed the use of sites like Sina Weibo as part of the learning experience will impact the volume of MOOC postings. Learners too may alternate between social media platforms to engage with other learners about their engagement in a MOOC(s). In the current setting in China, assessing user trends on MOOCs through social networking mechanisms will benefit from mining data from multiple sources.

\section{MOOC Activity during the week}

In a narrower sense, the activity patterns found in this study correlate with individuals' weekly routine. Yuan (2006) found that online students enrolled in distance education programs at East China Normal University tended to visit their course platform most frequently from Monday to Thursday (i.e., 600 student visits per day), with a noticeable drop on the weekend (i.e., 400 student visits per day).

The daily distribution of postings on MOOCs illustrates that, apart from the normal working hours during the daytime, a large number of users contribute MOOC postings in the later part of the evening. Findings also reveal that users are contributing MOOC postings at other intervals during the day. Two possibilities stem from this finding. One, more and more individuals are contributing postings using mobile phones, which are readily available and highly functional for microblogging. The other finding is that many users contributing MOOC postings are not working adults, but those who have greater flexibility in their work schedules, such as students. Clearly, further analysis is needed to discern the demographics of users in this study, as well as the rationale to rely on social networking to share information on MOOCs.

Most of all, content analysis is required to ascertain what users are talking about in regards to MOOCs, a topic for subsequent analysis with the data set used in this paper.

\section{Conclusions}

In this study, 95,015 MOOC tweets published by 62,074 users on the Sina Weibo platform between 2010 and 2015 were analysed to assess the public response to "the MOOC movement" in China. The time series of the data illustrate that MOOCs broke onto the educational scene as a new online teaching and learning phenomenon in China, resulting in the year 2013 receiving some recognition as being the Year of the MOOC in China, in contrast to the previous year where MOOCs was the buzzword in the US. The time series analyses revealed both predictable and surprising results. As noted, 2013 was a watershed year in regards to MOOCs in China and MOOC 
postings on the popular Sina Weibo platform reflected this interest, which continued into 2014. Deeper analyses revealed interesting patterns in how users on Sina Weibo contributed postings about MOOCs. The volume of postings reflected patterns that would be expected during a typical school year. The volume of postings declined during the summer and winter breaks, and increased dramatically immediately after these intervals. On a weekly basis, interest peaked on Monday, a finding that seems unusual considering this is the beginning of the work week and therefore a busy day for working adults. This trend gradually waned to Saturday before picking up again on Sunday. Finally, there appeared to be three distinct times during the day when the volume of postings was high. Evenings proved to be the most popular time frame when users were contributing postings on MOOCs.

Together, the analyses reveal potentially useful information for those who design and deliver MOOCs. The time of year to offer/not offer a MOOC, the time of week to release/not release new material, and the time of day to schedule/not schedule chat sessions, may provide insights that improve learner engagement in MOOCs, which tend to have high levels of attrition. Strategizing on timing is conducive to a relatively homogenous population, as is the case in China, where time zones and other factors lend itself to making somewhat accurate predictions on time. Other aspects on time could also be pondered and tested.

Finally, how to use microblogging, as an alternative to MOOC forums, to encourage engagement in MOOC learning is an important question that merits greater attention. While exploring how to harness the power of online delivery for educational needs, it is important to critically engage with an understanding of how learners actually interact online. This paper is one attempt to consider alternative mechanisms to engage in research on MOOCs with focus on learning populations in China.

\section{Notes}

1. The authors would like to thank the 12th five-year programme of the National Education Science Foundation (P. R. China) for financially supporting this research under Project No.ACA140009

2. Corresponding author: Dr. Kirk Perris, kirk.perris@bnu.edu.cn

3. One exception would be the several MOOCs that focus on learning Chinese, which cater to an international enrolment. Further, Chinese living abroad who are interested in learning are more incentivized to study in English or another functional language other than Chinese.

4. Few tweets $(n=83)$ were published between 2010 and 2011. To avoid noise in the data, these postings are excluded from analysis in this section. 


\section{References}

Barnes, C. (2013). MOOCs: The Challenges for Academic Librarians. Australian Academic \& Research Libraries, 44(3), 163-175. DOI:10.1080/00048623.2013.821048

Bollen, J., Mao, H., \& Zeng, X. J. (2010). Twitter mood predicts the stock market. CoRR, abs/ 1010.3003 .

China Education and Research Network. (1998). Action scheme for invigorating education towards the $21^{\text {st }}$ century. Retrieved from http://www.edu.cn/21st 1407/20060323/ t20060323 3996.shtml

Gao, F., Luo, T., \& Zhang, K. (2012). Tweeting for learning: A critical analysis of research on microblogging in education published in 2008-2011. British Journal of Educational Technology, 43(5), 783-801.

Guan, W., Gao, H., Yang, M., Li, Y., Ma, H., Qian, W. \& Yang, X. (2014). Analyzing user behaviour of the micro-blogging website Sina Weibo during hot social events. Physica A: Statistical Mechanics and its Applications, 395, 340-351.

Haklev, S. (2010). The Chinese national top level courses project: Using open educational resources to promote quality in undergraduate teaching. (Master's thesis, University of Toronto, Toronto, Canada). Retrieved from http://reganmian.net/top-level-courses/ Haklev Stian 201009 MA thesis.pdf

Jacquot, E. (2015). A primer on the evolving social media landscape of China, TechinAsia. Retrieved from https://www.techinasia.com/talk/primer-evolving-social-medialandscape-china/

Jordan, K. (2014). Initial trends in enrolment and completion of massive open online courses. The International Review Of Research In Open And Distributed Learning, 15(1).

Li, Q., \& Wang, T. (2012). MOOC: A giant open course model on the basis of Connectivism. Distance Education in China, 3, 30-36.

Ministry of Education. (2010). China's national plan for medium and long-term education reform and development. Retrieved from http://www.herrank.com/her/en/ newsdetail_55.htm

MOOC List. (2014). Entity's Country. Retrieved from https://www.mooc-list.com/countries

Pappano, L. (2012, November 2). The Year of the MOOC. The New York Times. 
Sadikov, E., Medina, M., Leskovec, J., \& Garcia-Molina, H. (2011, February). Correcting for missing data in information cascades. In Proceedings of the fourth ACM international conference on Web search and data mining (pp. 55-64). ACM.

Smith, C. (2015). By the numbers: 40 amazing weibo statistics. Expanded Ramblings. Retrieved from http://expandedramblings.com/index.php/weibo-user-statistics/

Universities UK. (2013). Massive open online courses: Higher education's digital moment? Retrieved from http://www.universitiesuk.ac.uk/highereducation/Documents/2013/ MassiveOpenOnlineCourses.pdf

van Treeck, T., \& Ebner, M. (2013). How useful is twitter for learning in massive communities? An analysis of two MOOCs. In K. Weller, A. Bruns, J. Burgess, M. Mahrt \& C. Puschmann (Eds.), Twitter \& Society (411-424). Bern, Switzerland: Peter Lang.

Wang, P. (2013). The new development and application of Massive online open course: From cMooc to xMooc. Modern Distance Education Research, 3, 13-19.

Wang, W. (2013). How MOOC affects higher education. Jiangsu Higher Education, 2, 53-57.

Wong. E. (2012, June 30). Test That Can Determine the Course of Life in China Gets a Closer Examination. New York Times. Retrieved from http://www.nytimes.com/2012/07/01/ world/asia/burden-of-chinas-college-entrance-test-sets-off-wide-debate.html? $\mathrm{r}=0$

Yuan, M. (2006). Research of the learning behavior of online learners. Zhe Jiang Modern Education Technology, 6(9).

Zhang, Z., Hong, L., \& Wen, Z. I. (2013). From OCW to MOOC: back to the origin of learning. Modern Distance Education Research, 3, 20-27.

Zhu, A. (2012, September 6). Massive Open Online Courses -- A Threat Or Opportunity To Universities? Forbes. Retrieved from http://www.forbes.com/sites/sap/2012/og/06/ massive-open-online-course-a-threat-or-opportunity-to-universities/

Zhang, K. (2015). Mining Data from Weibo to WeChat: A Comparative Case Study of MOOC Communities on Social Media in China. International Journal on E-Learning, 14(3), 305-329.

Zimmer, M. N., \& Proferes, J. (2014). A topology of Twitter research: Disciplines, methods, and ethics. Aslib Journal of Information Management, 6(3), 250-261. 
Public Response to "the MOOC Movement" in China: Examining the Time Series of Microblogging

Zhang, Perris, Zheng, and Chen

(C) Zhang, Perris, Zheng, and Chen

\section{Athabasca University $\mathbf{A}$}

(c) 